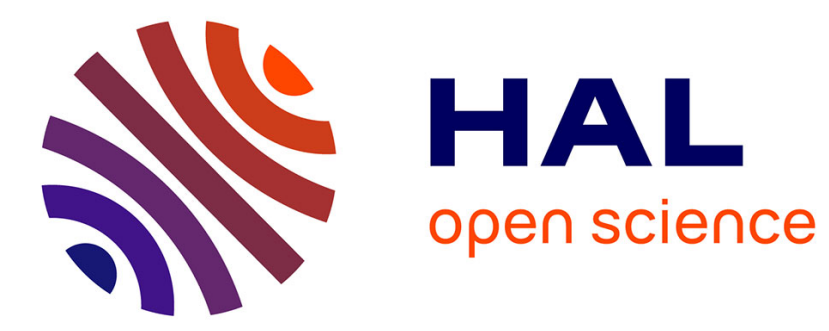

\title{
Fixed forced detection for fast SPECT Monte-Carlo simulation
}

Thomas Cajgfinger, Simon Rit, Jean Michel Létang, Adrien Halty, David Sarrut

\section{To cite this version:}

Thomas Cajgfinger, Simon Rit, Jean Michel Létang, Adrien Halty, David Sarrut. Fixed forced detection for fast SPECT Monte-Carlo simulation. Physics in Medicine and Biology, 2018, 63 (5), 10.1088/1361-6560/aa9e32 . hal-01692598

\section{HAL Id: hal-01692598 https://hal.science/hal-01692598}

Submitted on 6 Apr 2018

HAL is a multi-disciplinary open access archive for the deposit and dissemination of scientific research documents, whether they are published or not. The documents may come from teaching and research institutions in France or abroad, or from public or private research centers.
L'archive ouverte pluridisciplinaire HAL, est destinée au dépôt et à la diffusion de documents scientifiques de niveau recherche, publiés ou non, émanant des établissements d'enseignement et de recherche français ou étrangers, des laboratoires publics ou privés. 


\title{
Fixed Forced Detection for fast SPECT Monte-Carlo simulation
}

\author{
T. Cajgfinger ${ }^{1}$, S. Rit ${ }^{1}$, JM. Létang ${ }^{1}$, A. Halty ${ }^{1}$, and D Sarrut ${ }^{1}$ \\ ${ }^{1}$ Université de Lyon, CREATIS; CNRS UMR5220; Inserm U1044; INSA-Lyon; \\ Université Lyon 1; Centre Léon Bérard, France.
}

E-mail: david.sarrut@creatis.insa-lyon.fr

\begin{abstract}
.
Monte-Carlo simulations of SPECT images are notoriously slow to converge due to the large ratio between the number of photons emitted and detected in the collimator. This work proposes a method to accelerate the simulations based on Fixed Forced Detection (FFD) combined with an analytical response of the detector. FFD is based on a Monte-Carlo simulation but forces the detection of a photon in each detector pixel weighted by the probability of emission (or scattering) and transmission to this pixel. The method was evaluated with numerical phantoms and on patient images. We obtained differences with analog Monte-Carlo lower than the statistical uncertainty. The overall computing time gain can reach up to 5 orders of magnitude. Source code and examples are available in the Gate V8.0 release.
\end{abstract}

Keywords: Monte-Carlo simulations, SPECT, variance reduction

Submitted to: Phys. Med. Biol.

\section{Introduction}

Monte-Carlo simulations of SPECT images are useful for various applications such as designing imaging systems, investigating quantitative imaging or in Targeted Radionuclide Therapy (TRT). However, they are notoriously slow to converge due in particular to the large ratio between the number of photons emitted, denoted "primaries" in the following, and detected in the SPECT head (collimator + detector). Due to the solid angle of the detector and the loss of photons in the collimator, only one emitted photons in about $10^{4}$ reach the detector plane. This makes SPECT Monte-Carlo simulation a slow process, as every photon must be tracked and can generate secondary photons that should also be tracked through the detector. With "brute-force" Monte-Carlo methods, this can lead to very long simulation times, up to a few days for the simulation of a complete Siemens Symbia T system [1].

In order to decrease the computation time, several variance reduction techniques (VRT) have been developed [2]. The Geometrical Importance Sampling 
(GIS) [3] approach uses particle splitting and Russian roulette that consists in generating additional photons with a multiplicity value depending on the particle position, with the idea that the particles close to the detector have larger contributions than others. The speedup compared to analog simulation was estimated between 5 and 15. The Angular Response Function (ARF) [4, 1] is another acceleration method that computes detector response tables according to photons angles and energies $(\theta, \phi, E)$. Tables only need to be computed once, and are used during simulations to reduce tracking time into the SPECT head. The Forced Detection method (FD) [5] directs a particle towards the detector for every interaction. The particle is weighted according to the probability that a particle with such direction would exist. The acceleration compared to an analog simulation was estimated between 105 and 159. Multiple Projection Sampling (MPS) [5, 6] uses FD on several detectors at the same time and was up to 60 times faster than a regular FD Monte-Carlo. Convolution based Forced Detection (CFD) [6] uses FD with a Gaussian blurring kernel to spread the signal onto the image. It was recently used with SIMIND [7] for an estimated 6 orders of magnitude gain over analog Gate simulations. The main differences between the published methods and the one proposed in this article are the detector responses (ARF vs convolution) and the forced detection which, in our case, is performed for all detector pixels and take into account phantom specific attenuation along all paths, not only the path perpendicular to the detector. Finally, Fixed Forced Detection (FFD) was used for X-ray imaging $[8,9,10]$. It uses FD onto a set of points (all detector pixels or a reduced number) then uses interpolation for the other pixels.

Multiple platforms are used to simulate PET/SPECT images (SimSET [11], SIMIND [12], Geant4/GATE [13, 14]). Simulation System for Emission Tomography (SimSET) is a specialized package optimized for PET/SPECT simulations. It uses a photon history generator (PHG) to simulate photon interactions and transport inside a voxelized phantom. It contains several Variance Reduction techniques. GATE is widely used for SPECT simulations [15, 16], with modules available to perform photon tracking in a voxelized medium (generally obtained from a CT image), to manage a voxelised source of photons or any radionuclides, and to model the complete chain of the detector digitizer. It allows to generate different energy windows and it takes into account the energy resolution, the dead-time as well as the intrinsic spatial resolution of the detector. It has been shown to be accurate compared to experimental measures [17, 18]. Several VRT techniques described before (FD, MPS, CFD) were developed for GATE [5, 19]. However, these were mostly proofs of concept and are not available in GATE with the exception of ARFs. Monte-Carlo SPECT simulation of real SPECT imaging devices remains a complex and long computing process and its acceleration is still required. The purpose of this article was to investigate FFD for SPECT simulation which, to the best of our knowledge, was never investigated for this modality. 


\section{Materials and Methods}

The proposed method uses fixed forced detection for accelerating SPECT simulations. In SPECT imaging, photons are emitted by radionuclides inside the patient or the phantom. A fraction of the photons that escape the patient reach the SPECT head. The collimator intercepts most of the photons that are not perpendicular to the detector plane. The remaining photons are detected by a pixelated detector. Some scattered photons can still reach the detector plane and blur the image. Because photons lose energy during Compton scattering, detectors can be energy sensitive to separate the incoming photons into several energy windows: the primary window (around the radionuclide peak(s)) and the scatter window. The scatter window may be used to reduce the noise in the primary windows with Double or Triple Energy Window (DEW or TEW) scatter correction methods $[20,21]$. In the following, we first describe the FFD technique, then the link with the ARF method. We investigate the production of a single projection view here. All developments have been made within the GATE platform using Geant4 $10.2[14]$.

\subsection{Fixed Forced Detection}

The FFD method was initially published in $[8,9,10]$ for X-ray imaging. FFD is a variance reduction technique that forces photons towards each pixel of the detector for every Monte-Carlo interaction, which are of type $t \in$ \{decay, Rayleigh scattering, Compton scattering, fluorescence $\}$. These photons are given a weight that depends on their probability to reach the SPECT head and can be separated into a scattering and a transmission probability. The scattering probability is the probability for the photon to be directed towards the pixel, according to the interaction type $t$ of the photon. The transmission probability is the probability for the photon to reach the pixel, according to the traversed medium (Beer-Lambert law). The final detector count value $S_{j}$ for a pixel $j$ is the product of these probabilities for each interaction $i \in I$. The probabilities are computed under the assumption that the attenuation and the differential cross-section, which are computed at the center $\mathbf{p}_{j}$ of pixel $j$, are representative of all x-rays impinging the pixel surface. $S_{j}$ is given by Equation 1.

$$
S_{j}=\sum_{I} \Delta \Omega_{j}\left(\mathbf{x}_{\mathbf{i}}\right) \underbrace{\frac{1}{\sigma_{t_{i}}\left(E_{i}, Z_{i}\right)} \frac{\mathrm{d} \sigma_{t_{i}}}{\mathrm{~d} \Omega}\left(E_{i}, Z_{i}, \theta_{i j}\right)}_{\text {Scattering }} \underbrace{a\left(E_{i j}, \mathbf{x}_{i}, \mathbf{p}_{j}\right)}_{\text {Transmission }}
$$

where $\mathbf{x}_{\mathbf{i}}$ is the 3D-position at interaction $i, \sigma_{t}$ is the cross section of interaction type $t, \mathrm{~d} \sigma_{t} / \mathrm{d} \Omega$ is the differential cross-section that depends upon (1) the interaction type $t,(2)$ the energy $E_{i}$ of the photon before scattering, (3) the atomic number $Z_{i}$ of the material that triggered scattering and (4) the scattering angle $\theta_{i j} . E_{i j}$ is the 
energy after scattering. $a$ is the absorption from the interaction point to the pixel given by the the Beer-Lambert law Equation 2 where $\mu(E, \boldsymbol{x})$ is the $3 \mathrm{D}$ distribution of the energy-dependent linear attenuation coefficients.

$$
\begin{aligned}
& a(E, \mathbf{x}, \mathbf{p})=\exp \left(-\int_{0}^{1} \mu(E, \mathbf{x}+\alpha(\mathbf{p}-\mathbf{x})) \mathrm{d} \alpha\right) \\
& \Delta \Omega_{j}(\mathbf{s}) \approx \Delta S \frac{\left(\mathbf{s}-\mathbf{p}_{j}\right) \cdot \mathbf{n}}{\left\|\mathbf{s}-\mathbf{p}_{j}\right\|^{3}}
\end{aligned}
$$

In Equation 1, $\Delta \Omega_{j}(\mathbf{s})$ is the solid angle corresponding to pixel $j$ observed from point $\mathbf{x}_{i}$ and $\mathbf{p}_{j}$ the 3D spatial position of the center of the pixel $j$. We consider, that the distance between $\mathbf{x}_{i}$ and $\mathbf{p}_{j}$ is large enough to approximate the calculation of the solid angles using Equation 3, with the pixel surface $\Delta S$ and $\mathbf{n}$ the unit detector normal to the detector and oriented towards the source $\mathbf{s}$. Note that for a clinical SPECT, the distance is larger than a few centimeters while the pixel spacing is a few millimeters.

The distribution of linear attenuation coefficients $\mu(E, \mathbf{x})$ depends on the material definition, the physics list and the used cuts. It was computed with Geant 4 using the G4EmCalculator class for every material of the voxelised phantom. The differential cross sections $\mathrm{d} \sigma_{t} / \mathrm{d} \Omega$ used in Equation 1 were also retrieved from Geant4 using the G4CrossSectionHandler class. To implement Equation 1, a raycasting was performed from each interaction point towards all pixels of the detector. The ray-casting was discretized and performed using the reconstruction toolkit RTK [22] with Joseph's algorithm [23]. The ray-casting is independent for all pixels and was thus parallelized (multi-threaded CPU) for further computation gain. Note that all photons were forced with the FFD techniques, not only emitted primary photons but also all scattered ones, including multiple scattering.

\subsection{Linking FFD with Angular Response Function (ARF)}

The FFD method computes the probability for a photon to be directed towards a pixel and to reach it without further interaction. Two options were implemented to manage these photons upon reaching the detector. The first method passes photons outside of the volume back in the analog Monte-Carlo simulation with their respective directions, energies and weights. They are then tracked by the MonteCarlo engine. The second option links the output of the FFD directly to an ARF table [1]. It eliminates the creation of thousands of photons and further reduces the computation time. In the following, we consider this second option.

The ARF method uses Monte-Carlo simulations to compute a tabulated response function of the SPECT detector. It computes the probability of detection for incident photons depending on their energy $(E)$ and polar angles $(\theta, \phi)$ with the detector plane. Every photon reaching the SPECT head is stopped and a detection probability is stored into the pixel in the trajectory of the photon. This function 


\section{FFD SPECT simulation}

avoids photon tracking through the collimator and detector. Contrary to analog Monte-Carlo where most photons will be absorbed in the collimator (only around 1 in $10^{3}$ or $10^{4}$ emitted photons will be detected for standard SPECT systems), every incident photon will deposit with the ARF some weighted count and contribute to the image formation [1].

Computing the tabulated functions is a lengthy process: about $10^{9}$ to $10^{10}$ emitted primary photons are required to obtain a good statistical uncertainty for an energy window. For example, Figure 1 shows the case of a ${ }^{99 m}$ Tc source. The photon energy spectrum has a main $\gamma$-ray emission at $140.5 \mathrm{keV}(88.5 \%$ of decays). In this case, the digitizer is composed of two energy windows, the primary window $[126.45,154.55] \mathrm{keV}$ and the scatter window $[114,126] \mathrm{keV}$, sampled with $6.6 \mathrm{keV}$ steps, except for the $1 \mathrm{keV}$ step of the $140.5 \mathrm{keV}$ peak. Seven tables were computed for the ${ }^{99 \mathrm{~m}} \mathrm{Tc}$ windows.

Once the tables are computed, they can be used for every simulation having the same conditions (same collimator/detector and radionuclide energy windows), independently of the source distribution and the medium, phantom or patient. ARF tables require little disk space, in this case, around $57 \mathrm{MB}$ for 7 tables. When using another radionuclide, such as ${ }^{111} \mathrm{In}$, more tables are needed to keep the same energy resolution ( 6 for the $171 \mathrm{keV}$ peak, 8 for the $245 \mathrm{keV}$ peak and 4 for the scatter). In the following, we used the implementation proposed in Gate by Descourt et al. [1]. 


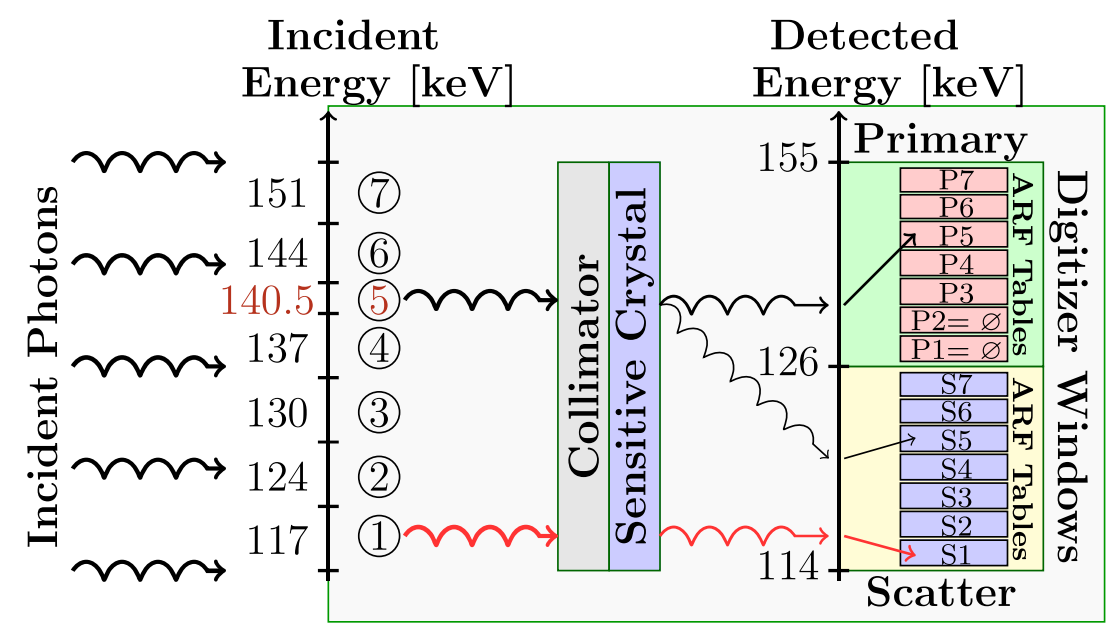

SPECT Head

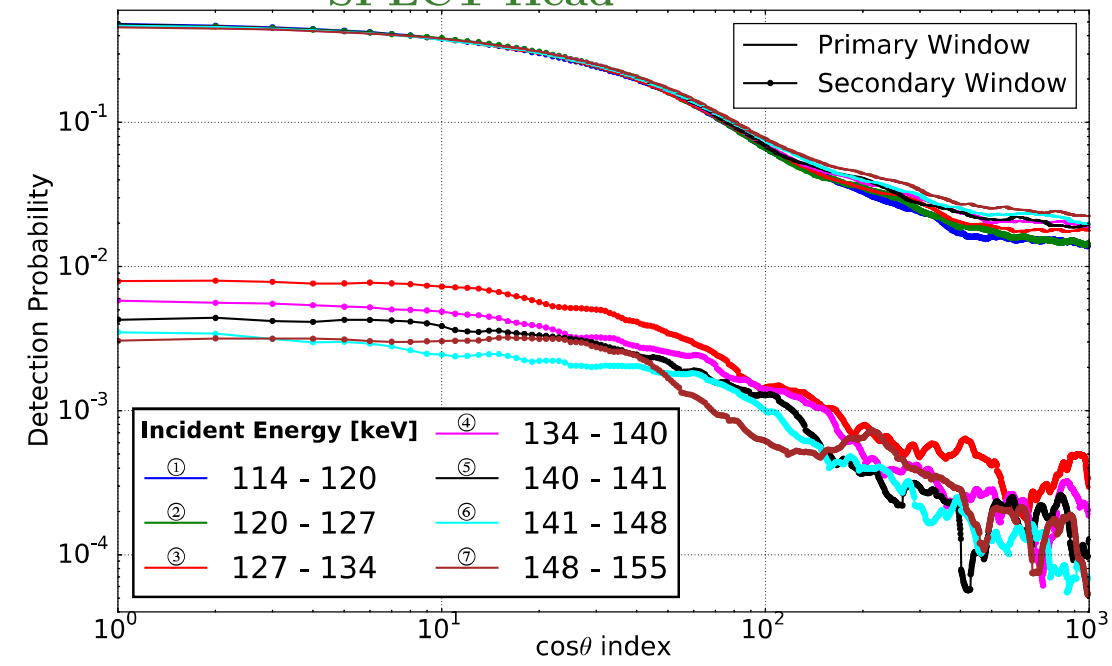

Figure 1. (Top) Schematic ARF table computation for a ${ }^{99} \mathrm{~m}_{\mathrm{Tc}}$ source. The digitizer windows are divided into small energy ranges of $7 \mathrm{keV}$ with smaller ones for the photo-peaks (indicated by (5) here). Two energy windows, primary and scatter, are shown, with the corresponding bins. (Bottom) ARF tables for ${ }^{99 \mathrm{~m}} \mathrm{Tc}$ with angle $\phi=0$. There are 5 curves for the primary window ( 1 and 2 are below the range) and 7 for the secondary window. We used the notation of [1]: the $\cos \theta$ index represents the binning of $\cos \theta$ (from 0 to 1 with 2048 bins). 


\section{Experiments}

The proposed method was compared to analog Monte-Carlo simulations, used as reference. The quality of the simulated images was assessed using the projection images and quantified using the Root Mean Square Distance (RMSD) defined as:

$$
\mathrm{RMSD}=\sqrt{\frac{\sum_{j=1}^{\mathrm{P}}\left(\bar{X}_{j}-\bar{X}_{j}^{\mathrm{ref}}\right)^{2}}{\mathrm{P}}}
$$

with $\bar{X}_{j}$ the sample mean value at pixel $j$ in the FFD image, $\bar{X}_{j}^{\text {ref }}$ the sample mean pixel value in the reference image obtained by analog Monte-Carlo, and $\mathrm{P}$ the number of pixels. The sample mean values were obtained by batch method [24]. $\mathrm{N}=20$ batches were used for all experiments. We used the normalized RMSD (NRMSD) defined as: RMSD $/ X^{\text {ref }}$, with $X^{\text {ref }}=\sum_{j} X_{j}^{\text {ref }}$. This criteria integrates both accuracy and precision and is limited by the relative statistical uncertainty $s$ of the reference Monte-Carlo. $s$ is estimated by $s=\sigma / X^{\mathrm{ref}}$, with $\sigma^{2}$ the variance averaged over the image $\frac{1}{n} \sum_{j} ! \sigma^{2}\left(X_{j}^{\text {ref }}\right)$. If $X_{j}$ in equation 4 were truly the unbiased mean, NRMSD would still be $s$. The variance $\sigma^{2}\left(X_{j}^{\text {ref }}\right)$ at pixel $j$ was obtained using:

$$
\sigma^{2}\left(X_{j}\right)=\frac{\sum_{b=1}^{\mathrm{N}}\left(X_{j}^{b}-\bar{X}_{j}\right)^{2}}{\mathrm{~N}(\mathrm{~N}-1)}
$$

where $\mathrm{N}$ is the number of batches, $X_{j}^{b}$ is the value of $X$ in batch $b$ in pixel $j$, and $\bar{X}_{j}$ is the mean value of $X$ in pixel $j$ evaluated over all batches.

The computation time was assessed by computing the simulation efficiency $\epsilon_{j}$ in each pixel $j$ :

$$
\epsilon_{j}=\left[t \times \sigma^{2}\left(X_{j}\right)\right]^{-1}
$$

where $t$ is the total simulation time, $\sigma^{2}\left(X_{j}\right)$ the variance in pixel $j$. Hence, the efficiency is increased if the time taken to obtain a given variance on the quantity of interest is reduced. The gain $G_{j}$ in a pixel $j$ is defined as the efficiency ratio between the two methods $\mathrm{m} 1$ and $\mathrm{m} 2: G_{j}=\epsilon_{j}^{\mathrm{m} 1} / \epsilon_{j}^{\mathrm{m} 2}$.

\subsection{First experiment: simple phantoms}

The first test $\mathrm{T}_{1}$ was the SPECT projection of an isotropic cubic source of $10 \mathrm{~cm}$ side of ${ }^{99 \mathrm{~m}}$ Tc inside a $20 \mathrm{~cm}$ cubic box. The box was either composed of air $\left(\mathrm{T}_{1 \mathrm{a}}\right)$ or water $\left(\mathrm{T}_{1 \mathrm{~b}}\right.$ and $\left.\mathrm{T}_{1 \mathrm{c}}\right)$. As we are interested in voxelized volumes, we considered the box as a matrix of $100^{3}$ voxels. The ${ }^{99 \mathrm{~m}}$ Tc source was modeled as a $140.5 \mathrm{keV}$ gamma source. The simulated SPECT device was one head of a GE Discovery 670 with a NaI crystal (field of view of $54 \mathrm{~cm} \times 40 \mathrm{~cm}$ ) equipped with a parallel-hole collimator. The Lowenergy High-resolution (LEHR) collimator, with holes diameter of $1.5 \mathrm{~mm}$ and holes 
height of $35 \mathrm{~mm}$, was modeled. In Gate, the digitizer module describes the global detector response and the signal processing chain after the gamma interactions in the crystal, replacing the PMTs that are not explicitly simulated [13]. We chose a spatial blurring of $5 \mathrm{~mm}$, and the two energy windows described above. The physics list used was emstandard_opt3 with production cuts for gamma equal to $0.1 \mathrm{~mm}$. The projections was defined with $100 \times 100$ pixels of $4 \mathrm{~mm}$ side.

\subsection{Second experiment: clinical data}

The second test $\mathrm{T}_{2}$ was a complete SPECT simulation with both a CT image and a voxelised activity source obtained from real clinical data. The data were gathered from a clinical trial that studied a labeled monoclonal antibody $(\mathrm{mAb})$, named ${ }^{90} \mathrm{Y}$ -OTSA101, targeting synovial sarcoma. In this trial, the dosimetry planning was performed from SPECT images acquired at several time point after ${ }^{111}$ In -OTSA101 injection. We selected one patient and a time integrated activity map was computed and used as a voxelised activity source [25]. The CT of the patient, resampled to $2 \times 2 \times 2 \mathrm{~mm}^{3}$, was used in the simulation. The ${ }^{99 \mathrm{~m}}$ Tc voxelized source was $4 \times 4 \times 4$ $\mathrm{mm}^{3}$. Figure 2 illustrates the input data.
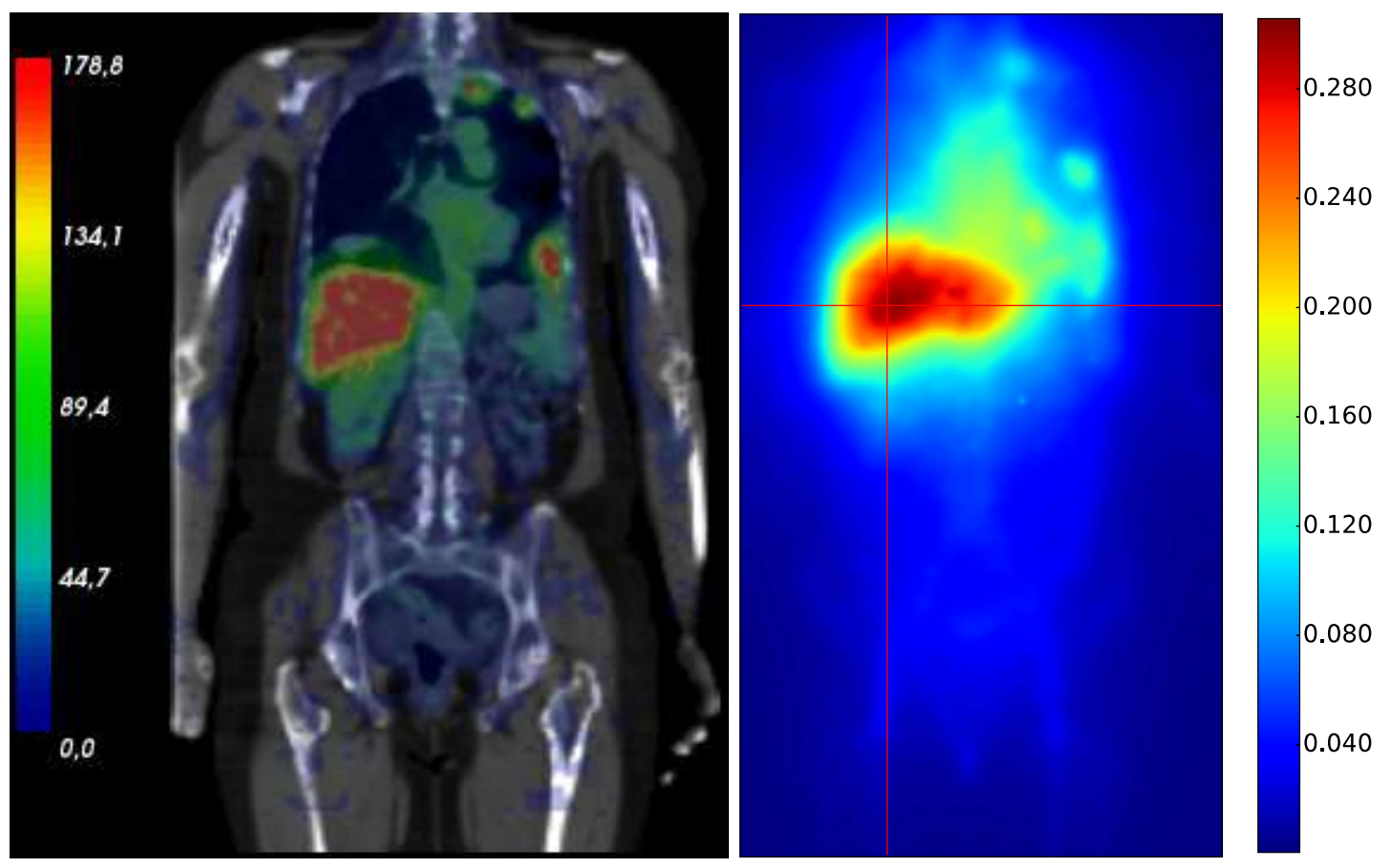

Figure 2. (Left) Activity map superimposed on a coronal slice of the CT scan; the scale indicates the number of counts in the voxels. (Right) FFD+ARF projection image obtained with $7 \times 10^{6}$ emitted primaries; the scale indicates the number of detected counts in the pixels. 


\section{Results}

\subsection{Test 1}

The accuracy of the FFD method was evaluated using NRMSD versus both analog Monte-Carlo and ARF. Figure 3 shows transverse profiles of simulated projection images for $\mathrm{T}_{1 \mathrm{a}}$ (in air, primary window), $\mathrm{T}_{1 \mathrm{~b}}$ (in water, primary window), $\mathrm{T}_{1 \mathrm{c}}$ (in water, scatter window). The numbers of emitted primaries for analog Monte-Carlo were $68 \times 10^{9}$ for $\mathrm{T}_{1 \mathrm{a}}$ and $\mathrm{T}_{1 \mathrm{~b}}$, and $165 \times 10^{9}$ for $\mathrm{T}_{1 \mathrm{c}}$. With FFD method, the numbers of emitted primaries were around $1 \times 10^{6}$. Those numbers have been chosen for practical reasons, leading to manageable computation times on the cluster. The use of equation 6 allows to compare them even if they do not lead to the same variance or detected counts.
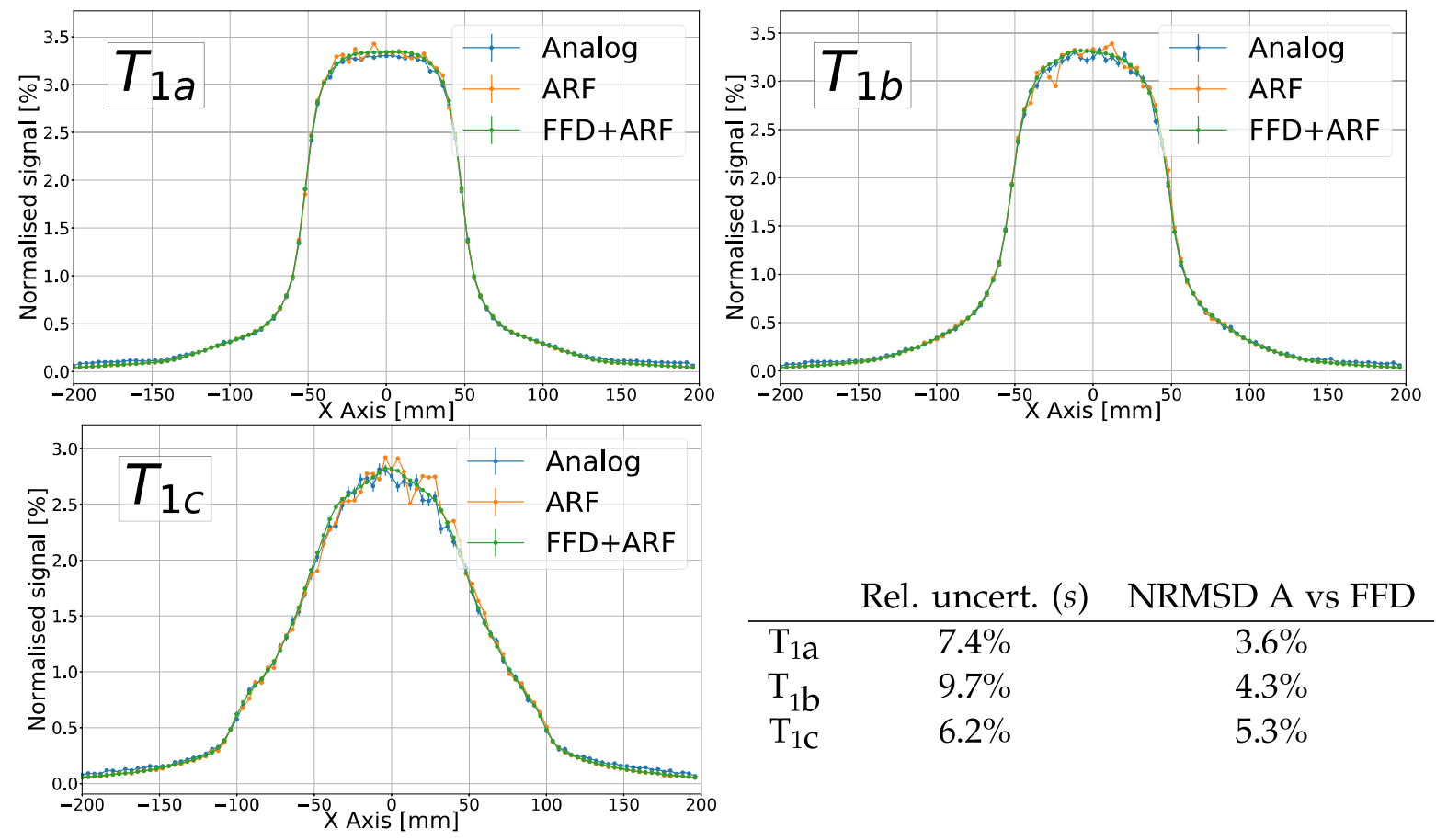

\begin{tabular}{ccc} 
& Rel. uncert. (s) & NRMSD A vs FFD \\
\hline $\mathrm{T}_{1 \mathrm{a}}$ & $7.4 \%$ & $3.6 \%$ \\
$\mathrm{~T}_{1 \mathrm{~b}}$ & $9.7 \%$ & $4.3 \%$ \\
$\mathrm{~T}_{1 \mathrm{C}}$ & $6.2 \%$ & $5.3 \%$
\end{tabular}

Figure 3. Comparison of the Analog, ARF and FFD+ARF profiles for the $10 \mathrm{~cm}$ cubic source in a $20 \mathrm{~cm}$ phantom (test 1). Left: phantom is composed of vacuum, primary window. Right: phantom is composed of water, primary window. Bottom: water phantom, scatter window. Relative statistical uncertainty of the reference MonteCarlo and NRMSD between analog and FFD are summarized in the table.

The shape of the profiles were very similar, with an NRMSD below $3.5 \%$ between analog and FFD+ARF methods. The efficiency of each method was computed with a batch method (20 simulations each) for $\mathrm{T}_{1 \mathrm{a}}, \mathrm{T}_{1 \mathrm{~b}}$ and $\mathrm{T}_{1 \mathrm{c}}$. The mean gains are given in Table 1 . Figure 4 shows the gains $G$ pixel by pixel. The total detected counts in the simulated images with analog Monte-Carlo were around 2, 4 and 8 millions counts respectively for $\mathrm{T}_{1 \mathrm{a}}, \mathrm{T}_{1 \mathrm{~b}}$ and $\mathrm{T}_{1 \mathrm{c}}$ which correspond to 
sensitivity around $3 \times 10^{-3}, 8 \times 10^{-3}$ and $4 \times 10^{-5}$. The approximate computing speed was around 1300-2000 tracked particles per seconds (PPS) for analog MonteCarlo while around only 35-50 PPS for FFD method (Intel Xeon CPU E5-1660 $3.30 \mathrm{GHz}$ ), obviously much slower due to the ray-casting.
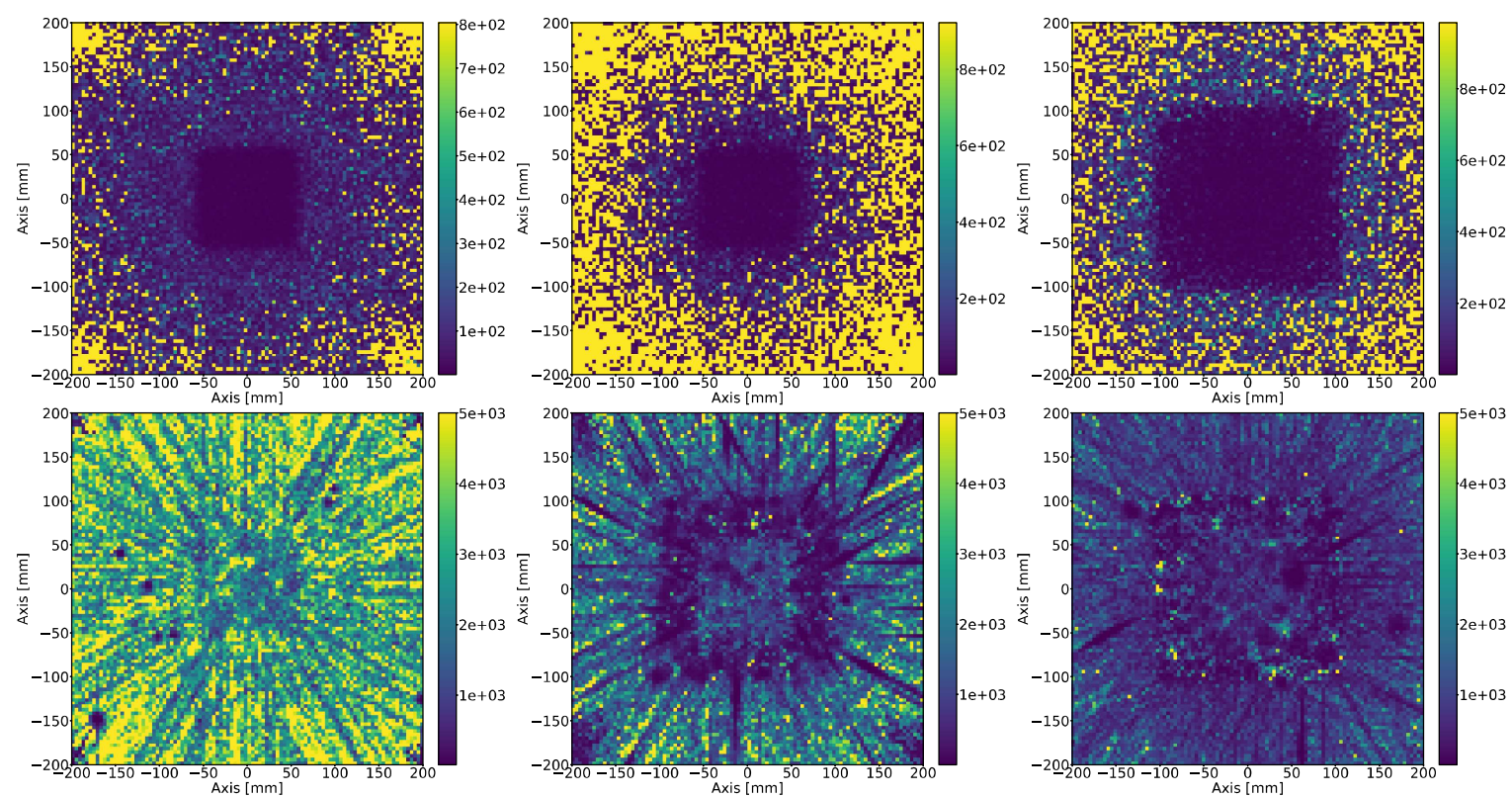

Figure 4. Gain map per pixel, the scales indicate the obtained acceleration factor $G$ between the two compared methods. Top: comparison between ARF and analog. Bottom: comparison between FFD+ARF and ARF. Left: $T_{1 a}$. Middle: $T_{1 b}$. Right: $\mathrm{T}_{1 \mathrm{c}}$.

\begin{tabular}{c|ccc} 
Test & Analog vs ARF & ARF vs FFD+ARF & Analog vs FFD+ARF \\
\hline $\mathrm{T}_{1 \mathrm{a}}$ & $1.7 \times 10^{2}$ & $3.5 \times 10^{3}$ & $5.2 \times 10^{5}$ \\
$\mathrm{~T}_{1 \mathrm{~b}}$ & $4.2 \times 10^{2}$ & $1.5 \times 10^{3}$ & $2.1 \times 10^{5}$ \\
$\mathrm{~T}_{1 \mathrm{c}}$ & $2.8 \times 10^{2}$ & $6.3 \times 10^{2}$ & $2.1 \times 10^{5}$
\end{tabular}

Table 1. Mean gain over the whole image for analog, ARF and FFD+ARF. Phantom filled with vacuum $\left(\mathrm{T}_{1 \mathrm{a}}\right)$ or water $\left(\mathrm{T}_{1 \mathrm{~b}}, \mathrm{~T}_{1 \mathrm{c}}\right)$, on the primary $\left(\mathrm{T}_{1 \mathrm{a}}, \mathrm{T}_{1 \mathrm{~b}}\right)$ or scatter window $\left(\mathrm{T}_{1 \mathrm{c}}\right)$.

\subsection{Test 2}

For $\mathrm{T}_{2}$, Figure 5 shows the transverse and longitudinal profiles of the simulated projection image in Figure 2 right. The numbers of emitted primaries were $4 \times 10^{10}$ (analog), $10^{10}$ (ARF) and $2 \times 10^{6}$ (FFD+ARF). The total computation time was 389 days for the analog Monte-Carlo, 87 days for ARF and 13 hours for FFD+ARF. The computations were performed on a cluster and converted to a total time for a single 
core (Intel Xeon CPU E5-1660 3.30GHz). The profiles follow similar shapes. For ten billion particles, the NRMSD on the central image region was $7.2 \%$ which can be compared to the relative statistical uncertainty of $6.3 \%$ in the same region. We therefore did not observe any bias of FFD in comparison to analog Monte-Carlo within the statistical uncertainty. The gain by pixel ranges from 1.3 to $11 \times 10^{3}$. The mean gain between ARF vs FFD+ARF methods for pixels with more than $20 \%$ of the maximum signal was 760 .
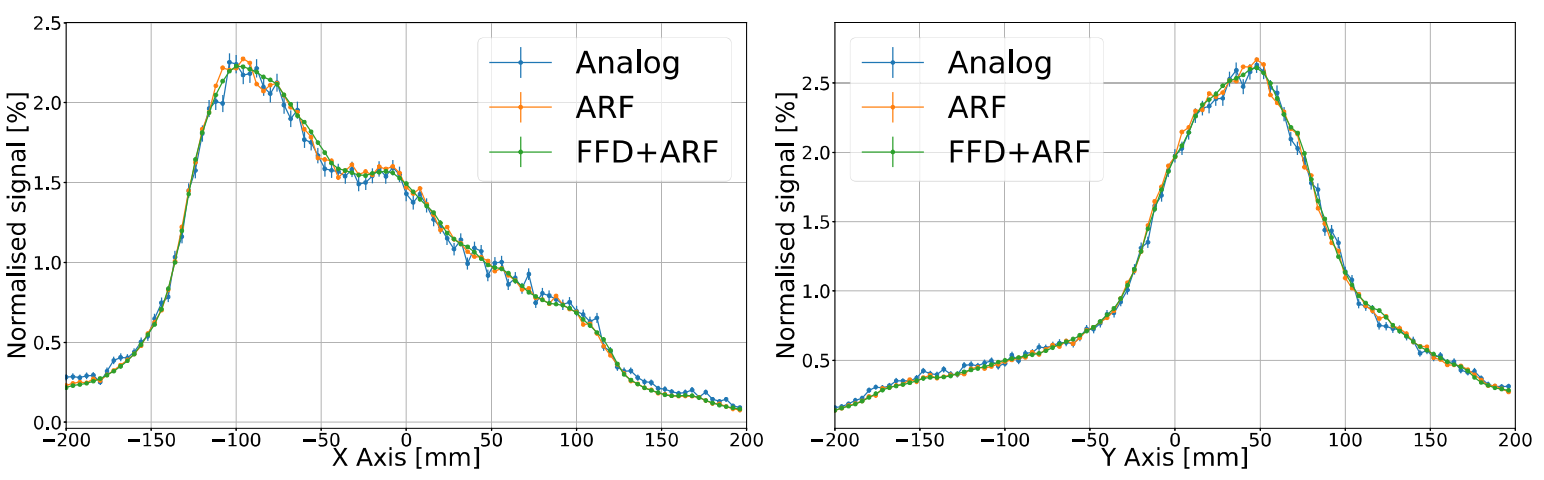

Figure 5. Transverse (left) and longitudinal (right) normalized profiles obtained on the simulated projected images of test 2 for the primary window. Profiles are shown for analog Monte-Carlo, ARF and FFD+ARF methods.

\subsection{Convergence study}

As already mentioned in previous works combining stochastic and deterministic strategies [26, 27], "hot spots" were observed when the statistic is not sufficient. Indeed, in a typical SPECT simulation, scattered particles are distributed in regions larger than the sources of emitting radionuclides. Therefore, the number of photons needed to get a representative sampling of the emitted particle's spatial distribution is larger for scatter than for primary photons. Because FFD allows for fast convergence of rare events, it can lead to bright spots before convergence. To clarify this phenomenon, simulations of a $10 \mathrm{~cm}$ edge size cubic source in a $20 \mathrm{~cm}$ cubic water phantom for different numbers of emitted primaries were computed. Resulting images were compared with the reference image obtained with $10^{7}$ emitted primaries. In order to illustrate the role of (i) the primaries, (ii) the source size relatively to the phantom and (iii) secondary photons, we performed the simulations with varying source sizes $(0.4,2,4,10,15$ and $20 \mathrm{~cm})$ and observed the differences in convergence speeds.

Figure 6 shows projection and RMSD images obtained with FFD+ARF for $10^{2}, 10^{4}$ and $10^{6}$ particles. Several "hot spots" can be observed due to the spatial distribution of emitted primaries. In the last image, we can see brighter spots inside the source with a smaller RMSD (0.04) than for the second image (0.225). 

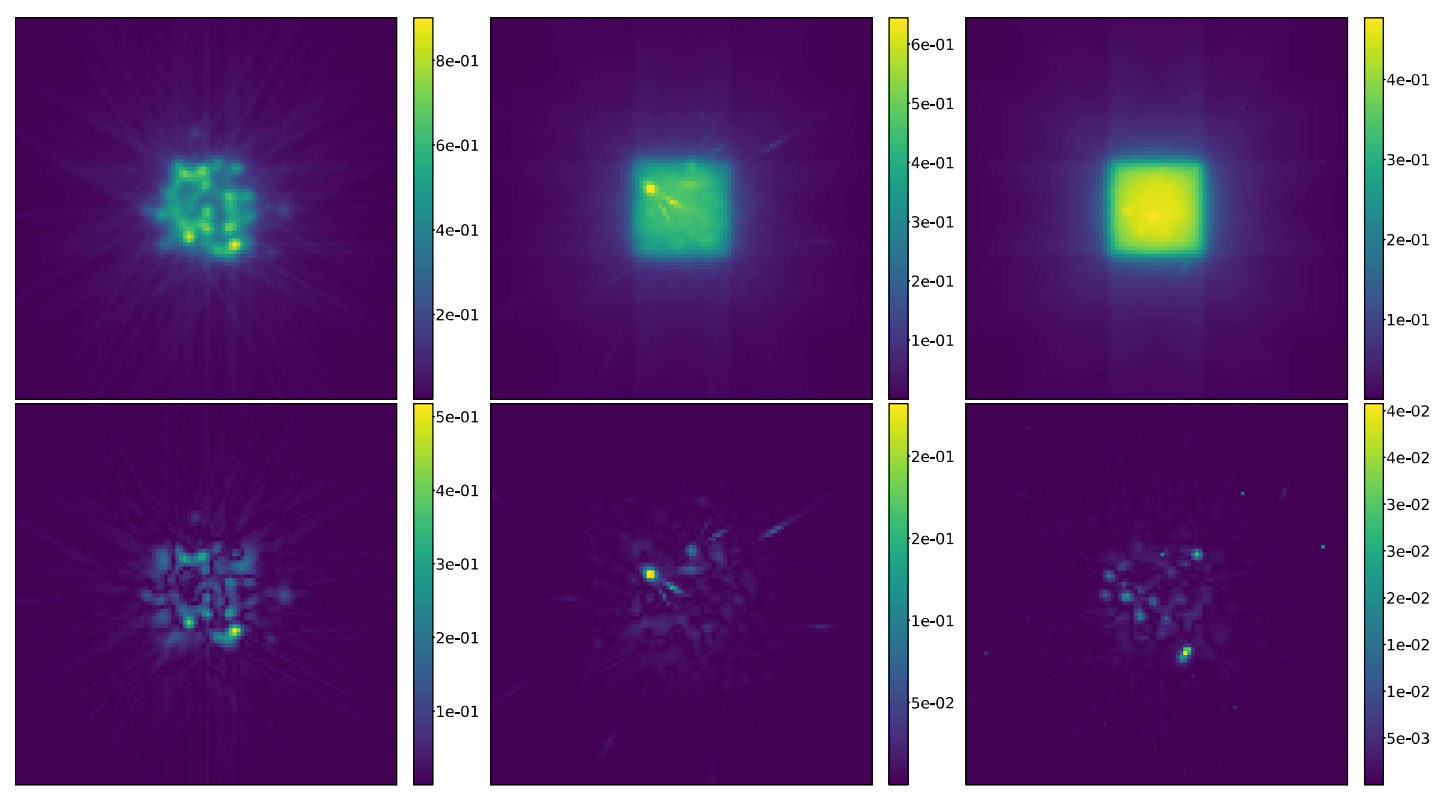

Figure 6. The top figures show SPECT projection images of a cubic source $(10 \mathrm{~cm})$ in a water phantom $(20 \mathrm{~cm})$ for different numbers of emitted primaries $\left(10^{2}, 10^{4}\right.$, $10^{6}$ ). The bottom figures show NRMSD between analog Monte-Carlo and FFD, in the same conditions.

Figures 7 (primaries only) and 8 (primaries and scattered photons) show precision and NRMSD according to the number of primaries $\left(10^{3}\right.$ to $\left.10^{6}\right)$ : precision (right image) was quantified by the percentage of pixels with a relative statistical uncertainty below $2 \%$. We choose $2 \%$ arbitrarily because this value this is a commonly chosen value in MC papers, but other threshold of interest may be used.
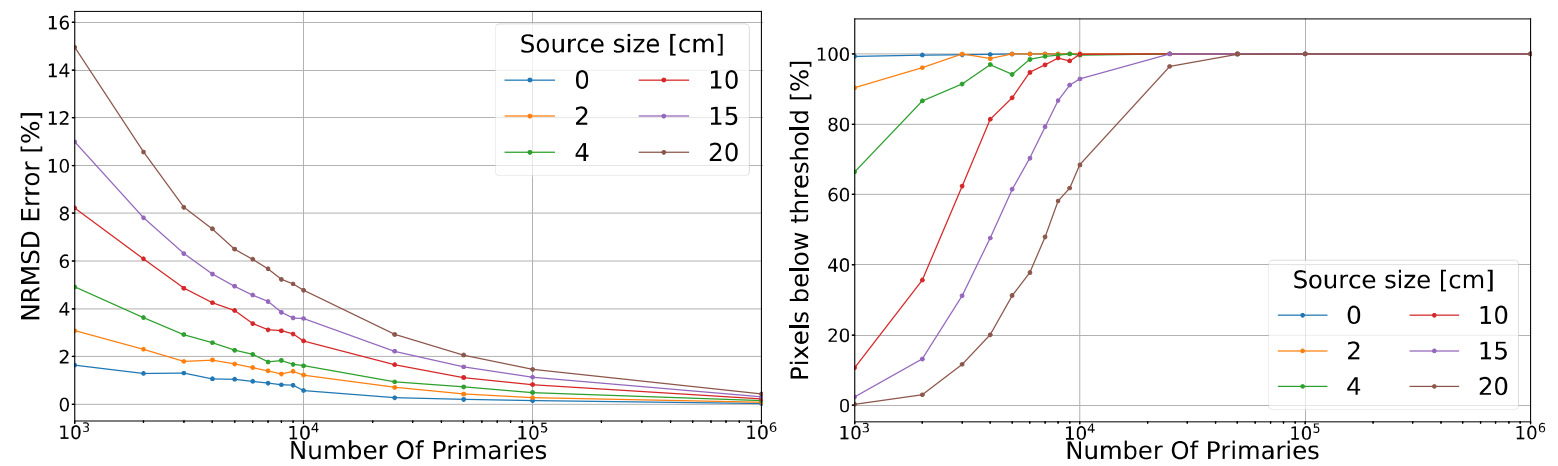

Figure 7. Evolution of the NRMSD (left) and percentage of pixels with a relative uncertainty below $2 \%$ (right) according to the number of emitted primaries $\left(10^{3}\right.$ to $10^{6}$ ), for primary photons only (not scatter).

For primary photons, both the pixel convergence and the NRMSD decreases were correlated with the size of the source. For a $4 \mathrm{~mm}$ source, only $10^{3}$ emitted primaries were needed to get less than $2 \%$ NRMSD and $>95 \%$ pixel convergence, while $10^{5}$ were required to obtain similar results with a source as large as the 

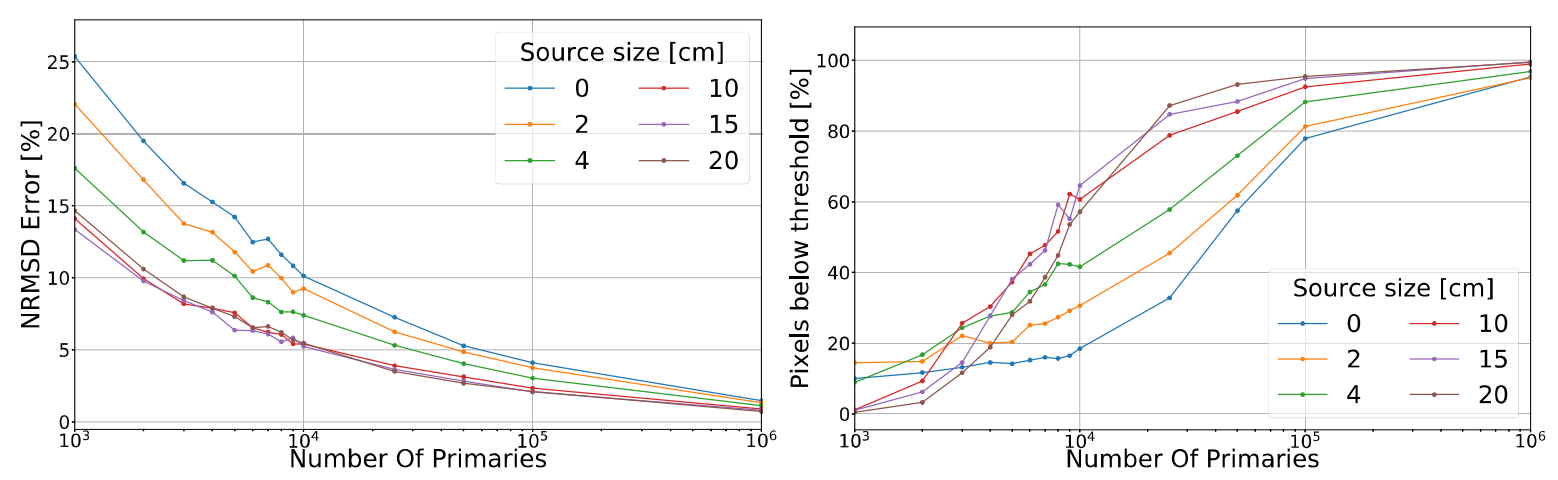

Figure 8. Evolution of the NRMSD (left) and percentage of pixels with a relative uncertainty below $2 \%$ (right) according to the number of emitted primaries $\left(10^{3}\right.$ to $10^{6}$ ), for both primary and scattered photons.

phantom $(20 \mathrm{~cm})$. When taking scattered photons into account, voxels that are far from the source will have a low photon count, thus larger sources meant a quicker convergence.

\section{Discussion}

The FFD method reached very good agreement to analog simulations for simple cases $\left(T_{1 a}, T_{1 b}, T_{1 c}\right)$ as well as for realistic cases $\left(T_{2}\right.$ patient data). NRMSD values were always in the order of the relative statistical uncertainty, which indicates that the bias is much smaller than the Monte-Carlo uncertainty. Regarding efficiency, the gains were up to $10^{3}$ compared to ARF only and $10^{5}$ compared to analog. Also, as already pointed out by Song et al original ARF paper [4], the detection probability of gamma with incident angle larger than 15-20 degrees is close to zero (excepted for high energy gamma). Thus, additional time gain could be reached if gamma with large incident angle are ignored during the FFD step. With other types of collimators, for example for high-energy radionuclide like ${ }^{131} \mathrm{I}$, efficiency may change slightly but it has not been investigated here. Both the NRMSD and the statistical uncertainty were dependent on the size of the source. For a $2 \mathrm{~cm}$ source, only $3 \times 10^{3}$ emitted primaries were needed to obtain more than $95 \%$ of the pixels below $2 \%$ uncertainty and less than $2 \%$ NRMSD error, while $5 \times 10^{4}$ emitted primaries were needed when the source was larger $(20 \mathrm{~cm})$. A point source needs only one emitted primary to generate the projection image of detected primaries. When scattered photons are considered, the results were reversed: for a $20 \mathrm{~cm}$ source, $10^{5}$ emitted primaries are needed to reach a NRMSD below $2 \%$ for $95 \%$ of the pixels, while $10^{6}$ were needed for a smaller $2 \mathrm{~cm}$ source. In total, scattered photons represent in the water phantom $26 \%$ (23.8\% Compton, $2.5 \%$ Rayleigh) of the total signal. Also note that, because of the proposed variance reduction, the obtained image will not contain a noise similar to the analog Monte-Carlo method. 
If the user wants to reproduce it, the data should be scaled to the correct number of counts and be corrupted with Poisson noise.

Finally, we did not investigate a practical criterion to determine convergence because it mostly depends of the application and will be very different if user is interested in detecting the primary signal or the scattered one in a particular region of the image. In the proposed implementation, only one voxelised volume can be used in a simulation and no other volumes could be positioned between this volume and the ARF plane.

\section{Conclusion}

We propose a method to reduce the variance of Monte-Carlo SPECT simulations using FFD in order to get a better computation time. This implementation was further improved by linking the FFD to ARF tables. The measured gain versus analog simulations was up to 5 orders of magnitude for realistic patient simulations. For a relative statistical uncertainty below $2 \%$, this method can simulate a projection in less than 2 hours on a single core instead of days. Only a few simulations cases have been tested, further studies are needed for further adoption. This method with examples and documentation is available in the last Gate V8.0 version.

\section{Acknowledgments}

This work was partly supported by t-Gate ANR-14-CE23-0008, the SIRIC LYric Grant INCa-DGOS-4664 and the LABEX PRIMES (ANR-11-LABX-0063/ ANR-11-IDEX0007).

\section{References}

[1] P. Descourt, T. Carlier, Y. Du, X. Song, I. Buvat, E. C. Frey, M. Bardiès, B. M. W. Tsui, and D. Visvikis. Implementation of angular response function modeling in SPECT simulations with GATE. Physics in Medicine and Biology, 55(9):N253, 2010.

[2] David R Haynor, Robert L Harrison, and Thomas K Lewellen. The use of importance sampling techniques to improve the efficiency of photon tracking in emission tomography simulations. Medical Physics, 18(5):990-1001, 1991.

[3] J. De Beenhouwer. Acceleration of GATE Monte Carlo Simulations. âĂć, Gent University, âĂć, âĂć 2009. âĂć.

[4] X Song, W P Segars, Y Du, B M W Tsui, and E C Frey. Fast modelling of the collimator-detector response in monte carlo simulation of spect imaging using the angular response function. Physics in medicine and biology, 50:1791-1804, April 2005.

[5] J. De Beenhouwer, S. Staelens, S. Vandenberghe, and I. Lemahieu. Acceleration of GATE SPECT simulations. Medical Physics, 35(4):1476-1485, 2008.

[6] S. Liu, M. A. King, A. B. Brill, M. G. Stabin, and T. H. Farncombe. Accelerated SPECT Monte Carlo simulation using multiple projection sampling and convolution-based forced detection. IEEE Transactions on Nuclear Science, 55(1):560-567, Feb 2008. 
[7] M. I. Karamat and T. H. Farncombe. A comparison between gate and accelerated convolutionbased forced detection simind for low- and medium-energy collimators: A simulation study. IEEE Transactions on Radiation and Plasma Medical Sciences, 1(1):36-45, Jan 2017.

[8] A.P. Colijn and F.J. Beekman. Accelerated simulation of cone beam x-ray scatter projections. IEEE Transactions on Medical Imaging, 23(5):584-590, 2004.

[9] N. Freud, J.-M. Létang, and D. Babot. A hybrid approach to simulate multiple photon scattering in x-ray imaging. Nuclear Instruments and Methods in Physics Research Section B: Beam Interactions with Materials and Atoms, 227(4):551 - 558, 2005.

[10] G. Poludniowski, P. M. Evans, V. N. Hansen, and S. Webb. An efficient Monte Carlo-based algorithm for scatter correction in $\mathrm{keV}$ cone-beam CT. Physics in Medicine and Biology, 54(12):3847, 2009.

[11] R. L. Harrison, S. D. Vannoy, D. R. Haynor, S. B. Gillispie, M. S. Kaplan, and T. K. Lewellen. Preliminary experience with the photon history generator module of a public-domain simulation system for emission tomography. In 1993 IEEE Conference Record Nuclear Science Symposium and Medical Imaging Conference, pages 1154-1158, Oct 1993.

[12] Michael Ljungberg and Sven-Erik Strand. A Monte Carlo program for the simulation of scintillation camera characteristics. Computer Methods and Programs in Biomedicine, 29(4):257 272, 1989.

[13] S. Jan, D. Benoit, E. Becheva, T. Carlier, F. Cassol, P. Descourt, T. Frisson, L. Grevillot, L. Guigues, L. Maigne, C. Morel, Y. Perrot, N. Rehfeld, D. Sarrut, D. R. Schaart, L. Stute, U. Pietrzyk, D. Visvikis, N. Zahra, and I. Buvat. GATE V6: a major enhancement of the GATE simulation platform enabling modelling of CT and radiotherapy. Physics in Medicine and Biology, 56(4):881, 2011.

[14] D. Sarrut, M. Bardiès, N. Boussion, N. Freud, S. Jan, J. M. Létang, G. Loudos, L. Maigne, S. Marcatili, T. Mauxion, P. Papadimitroulas, Y. Perrot, U. Pietrzyk, C. Robert, D. R. Schaart, D. Visvikis, and I. Buvat. A review of the use and potential of the gate monte carlo simulation code for radiation therapy and dosimetry applications. Medical Physics, 41(6), 2014.

[15] S. V. Spirou, P. Papadimitroulas, P. Liakou, P. Georgoulias, and G. Loudos. Investigation of attenuation correction in SPECT using textural features, Monte Carlo simulations, and computational anthropomorphic models. Nucl Med Commun, 36(9):952-961, Sep 2015.

[16] A. Konik, M. T. Madsen, and J. J. Sunderland. GATE simulations of small animal SPECT for determination of scatter fraction as a function of object size. IEEE Transactions on Nuclear Science, 59(5):1887-1891, Oct 2012.

[17] K. Assie, A. Dieudonne, I. Gardin, P. Vera, and I. Buvat. A preliminary study of quantitative protocols in Indium 111 SPECT using computational simulations and phantoms. IEEE Transactions on Nuclear Science, 57(3):1096-1104, June 2010.

[18] D. Osborne S. Lee, J. Gregor. Development and validation of a complete GATE model of the Siemens Inveon trimodal imaging platform. Molecular Imaging, Oct 2013.

[19] Jan De Beenhouwer, Steven Staelens, M Dressel, Yves D'Asseler, Stefaan Vandenberghe, Ignace Lemahieu, and Rik Van de Walle. Geometrical importance sampling and pulse height tallies in gate. In Engineering in Medicine and Biology Society, 2004. IEMBS'04. 26th Annual International Conference of the IEEE, volume 1, pages 1349-1352. IEEE, 2004.

[20] I Buvat, H Benali, A Todd-Pokropek, and R Di Paola. Scatter correction in scintigraphy: the state of the art. European Journal of Nuclear Medicine, 21(7):675-694, 1994.

[21] Brian F Hutton, Irene Buvat, and Freek J Beekman. Review and current status of spect scatter correction. Physics in medicine and biology, 56(14):R85, 2011.

[22] S. Rit, M. Vila Oliva, S. Brousmiche, R. Labarbe, D. Sarrut, and G. C. Sharp. The reconstruction toolkit (RTK), an open-source cone-beam CT reconstruction toolkit based on the Insight toolkit (ITK). Journal of Physics: Conference Series, 489(1):012079, 2014.

[23] P. M. Joseph. An improved algorithm for reprojecting rays through pixel images. IEEE Transactions on Medical Imaging, 1(3):192-196, Nov 1982. 
[24] B. R. B. Walters, I. Kawrakow, and D. W. O. Rogers. History by history statistical estimators in the BEAM code system. Medical Physics, 29(12):2745-2752, 2002.

[25] D. Sarrut, JN. Badel, A. Halty, G. Garin, D. Perol, P. Cassier, JY. Blay, D. Kryza, and AL. Giraudet. 3D absorbed dose distribution estimated by Monte Carlo simulation in radionuclide therapy with a monoclonal antibody targeting synovial sarcoma. EJNMMI Phys, to appear, 2017.

[26] F. Smekens, N. Freud, J. M. Létang, J-F. Adam, C. Ferrero, H. Elleaume, A. Bravin, F. Estève, and D. Babot. Simulation of dose deposition in stereotactic synchrotron radiation therapy: a fast approach combining Monte Carlo and deterministic algorithms. Phys Med Biol, 54(15):46714685, Aug 2009.

[27] F. Smekens, J. M. Létang, C. Noblet, S. Chiavassa, G. Delpon, N. Freud, S. Rit, and D. Sarrut. Split exponential track length estimator for monte-carlo simulations of small-animal radiation therapy. Physics in Medicine and Biology, 59(24):7703, 2014. 